\title{
Detached Eddy Simulation for Turbulent Flows in a Pipe with a Snowflake Fractal Orifice
}

\author{
H.W. Zheng, F.C.G.A. Nicolleau, and N. Qin
}

\begin{abstract}
Turbulent flows in a pipe with a snowflake fractal shape (SF2) orifice are investigated using the parallelized, density-based, dynamic mesh and detached eddy simulation code (DG-DES) (Xia, PhD thesis, 2005; Xia and Qin, AIAA 2005$106,2005)$. For comparison with the laboratory experiment, the flow is essentially a low Mach number flow. In order to tackle the low speed problem of the density based method, the SLAU (Simple Low dissipation AUSM) (Shima and Kitamura in 47th AIAA Aerospace Sciences Meeting including the New Horizons Forum and Aerospace Exposition, AIAA 2009-136, 2009) is adopted in this paper. The scheme exhibits low numerical dissipations for low speeds and needs no problem-dependent "cut-off Mach number". The results for the flows after the orifice are compared with those of the corresponding experiment (Chong, PhD thesis, 2008). Comparisons show good agreements in the mean velocity profiles at the different holes.
\end{abstract}

\section{Introduction-Motivation}

Turbulent flows can be found in many industrial processes but remain hard to tackle. The main tools that have been proposed to understand fluid turbulence are spectral and to a lesser extend wavelet analyses. They have shown their limits, mainly because they were just used as post-processing tools, and we are still lacking a good understanding of the structure of turbulent flows and how they work. Fractal anal-

H.W. Zheng $(\bowtie)$

LHD, Institute of Mechanics, Chinese Academy of Sciences, Beijing 100190, China

e-mail: h.zheng@imech.ac.cn

F.C.G.A. Nicolleau

Sheffield Fluid Mechanics Group, Department of Mechanical Engineering, The University

of Sheffield, Mappin Street, Sheffield S1 3JD, UK

e-mail: F.Nicolleau@sheffield.ac.uk

N. Qin

Department of Mechanical Engineering, The University of Sheffield, Mappin Street, Sheffield S1

3JD, UK

e-mail: N.Qin@ sheffield.ac.uk

F.C.G.A. Nicolleau et al. (eds.), New Approaches in Modeling Multiphase Flows and 
yses have been around for a while in fluid mechanics but were not until recently really related to the physics of turbulence [11] or were used as well more as a postprocessing tool $[14,22,23]$ giving interesting interpretations and quantifications of mixing or interface but links with turbulence properties were difficult to establish.

Recently a different approach has been adopted by $[7,15,17]$. Rather than trying to understand turbulence 'ex nihilo' from the Navier Stokes equations or describing the flow as an external observer, these new approaches are relying on attempts at interacting with it from within. Although the physics of turbulent flows is still not well understood, one property we can be sure of is the multi-scale nature of turbulent flows. So, what better interaction there can be than to interact with turbulence in a fractal manner; either through a fractal object or a numerical fractal forcing. This new way of thinking gave rise to many different experiments, see [9] for a good summary of fractal activities in fluid mechanics. To be complete, KS's ${ }^{1}$ new developments need also to be cited here as participating in that new trend at looking at the structure of turbulent flows through fractal analysis. Though KS are not real flows they have benefited from, and in some cases paved the way for, these recent developments, in particular in terms of straining eddying regions [5] or stagnation points which are the backbone of KS accuracy and have been investigated in terms of fractal distribution (see e.g. [4, 6, 24]).

We do not want to go into details in trying to summarize all these studies exhaustively here, our contribution is of a different kind at this stage: more practical than these fundamental works on turbulence. We do not actually expect to contribute to this new understanding of turbulence yet but rather more modestly want to use it. At the same time fractal approaches were taking a new direction, new computational methods were developing as well. While physicists were trying to understand more deeply how turbulence works, engineers had to tackle more and more complex geometries and problems. Old numerical schemes as Reynolds Average Navier Stokes (RANS) were not doing the job and LES or DNS were still (and are to be for a long time) too time and memory consuming. Detached Eddy Simulations (DES) emerged in this context. They were rather empirical in their construction (some would say rather unphysical) wanting the best from both RANS and LES. By contrast to RANS, DES give a richer, more detailed (less averaged) picture of the flow field but give rise to new problems, how to interface RANS and LES is at the core of all of them. This inspired us to use DES to investigate turbulence flows in a pipe with multi-scale fractal-shaped orifices. The use of a pipe flow was in part driven by engineering applications where multi-scale orifices are known to give more accurate flowmetres. This observation is empirical and no link with fractal geometry has been proposed so far. In longer term we hope to understand better the physics underlying this observation [1].

There have been numerical simulations of flows through or around fractals or fractal forcing $[10,12,13]$. However, the Reynolds numbers achieved in DNS can not be very high in contrast to laboratory experiments. Hence, in [10] the numerical

\footnotetext{
${ }^{1}$ We direct the reader to other papers in this Book Series for details on Kinematic Simulation (KS).
} 
Fig. 1 Flow setting

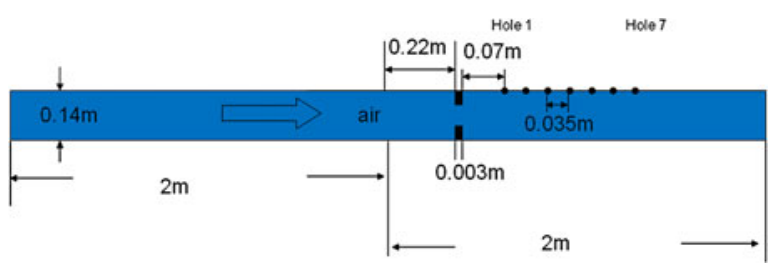

results could only be compared qualitatively with experimental results. These comparisons were however encouraging showing the need for lower Reynolds experiments and higher Reynolds computations. The former is much easier than the later and there is need for methods aiming at the modeling of fractal flows. Apart from DNS, there is one paper [2] on a high-Reynolds number flow over a tree-like fractal using large eddy simulation (LES). A renormalized numerical simulation (RNS) was needed to model the drag of the unresolved branches of the fractal tree. The shortcomings of numerical schemes when it comes to model fractals are mentioned in [9]: all closures rely somehow on a hypothesis of isotropy below certain scales. DES are no exception but because of their hybrid nature (combination of RANS and LES-DNS) are much more versatile than other methods and perhaps amendable because one may be able to limit the closure problem to particular regions of space and use DNS were it is vital for accurate predictions.

We present preliminary results here in order to validate our numerical scheme and eventually assess the feasibility of studying the turbulent flows after fractal orifices by the detached eddy simulation technique (DES [19, 21]). We start from a simple problem with a fractal star based on von Koch's snowflake. The numerical experiment is combined with a laboratory experiment reproducing the same conditions $[1,3]$. The experimental set up is shown in Fig. 1. We expect later on to increase step by step the complexity of the fractal keeping the same fractal dimension. Thus, we do not try to keep the area constant, again our purpose is to test the reaction of the DES model to the forcing of new scales, so our plan is to keep the fractal dimension constant and add new scales step by step. The results presented here are for the simple grid presented in Fig. 2 and are a first validation of the DES code we are going to use.

The simulation is based on an density-based dynamic mesh and the detached eddy simulation code (DG-DES) developed by [26, 27] for solving the unsteady compressible Favre-averaged Navier-Stokes equations and the Spalart and Allmaras (S-A) equation [20]. It is parallelized by using the Message Passing Interface (MPI) library and the unstructured mesh partitioning tool METIS [8]. In order to tackle the low Mach flow, the scheme is replaced by a low dissipation upwind SLAU (Simple

Fig. 2 The fractal orifice SF2 based on von Koch's curve

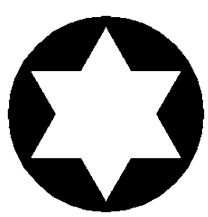


Low dissipation AUSM) scheme [18]. The results are compared with the corresponding experimental results done in $[1,3]$.

The paper is organized as follows: the governing equations and numerical method are simply described respectively in the second and third sections. After that, the method is applied to investigate the turbulent flow after a snowflake fractal orifice (SF2). The results are presented in the fourth section.

\section{Governing Equations}

The flows are solved by using a density-based finite volume solver using a detached eddy simulation (DG-DES). It is known that DES [19,21] is a combination of Large Eddy Simulations (LES) and Reynolds Average Navier-Stokes methods (RANS). Its main feature is that it can be automatically switched to a sub-grid scale (SGS) model in the LES regions and to a RANS model in the RANS regions by modifying a distance function.

For density-based methods, the flow is modeled using the unsteady compressible Favre-averaged Navier-Stokes equations,

$$
\partial_{t} \int_{\Omega} \mathbf{U} d V+\int_{S} \mathbf{F} d S=0
$$

with the flux vector $\mathbf{F}=\mathbf{F}^{c}-\mathbf{F}^{v}$,

$$
\mathbf{F}^{c}=(\mathbf{u} \cdot \mathbf{n}) \mathbf{U}+p\left(\begin{array}{c}
0 \\
\mathbf{n} \\
\mathbf{u} \cdot \mathbf{n}
\end{array}\right), \quad \mathbf{F}^{v}=\left(\begin{array}{c}
0 \\
{[\tau]} \\
{[\tau] \bullet \mathbf{u}+\mathbf{q}}
\end{array}\right) \cdot \mathbf{n}
$$

Here, $\mathbf{U}$ is the state vector $(\rho, \rho \mathbf{u}, E)^{T}, \rho$ is the fluid density, $\mathbf{u}$ is the flow velocity, $p$ is the static pressure, $E$ is the total energy $\left(E=\rho e+0.5 \rho \mathbf{u}^{2}\right), \mathbf{n}$ is the unit normal direction of the surface, and $[\tau]$ is the viscous stress tensor,

$$
[\tau]=\left(\mu_{L}+\mu_{\text {tur }}\right)\left[\nabla \mathbf{u}+\nabla^{T} \mathbf{u}-\frac{2}{3}(\nabla \cdot \mathbf{u})[\mathbf{I}]\right] .
$$

The heat flux $\mathbf{q}$ is approximated by the linear relationship

$$
\mathbf{q}=\left(\kappa_{L}+\kappa_{t u r}\right) \nabla T
$$

where the thermal conduction coefficient is expressed by

$$
\kappa=\kappa_{L}+\kappa_{t u r}=\frac{\mu_{L} c_{p}}{\operatorname{Pr}_{L}}+\frac{\mu_{t u r} c_{p}}{\operatorname{Pr}_{t u r}}
$$

in which $c_{p}$ is the specific heat at constant pressure, $\operatorname{Pr}_{L}$ and $\operatorname{Pr}_{t u r}$ are the Prandtl numbers.

To close the system, the turbulence viscosity in Eq. (3) is calculated by

$$
\mu_{t u r}=f_{v 1} \rho \tilde{v}
$$

with the damping function, 


$$
f_{v 1}=\frac{\chi^{3}}{\chi^{3}+C_{v 1}^{3}}, \quad \chi=\frac{\tilde{v}}{v_{L}} .
$$

The modified viscosity $\tilde{v}$ is obtained by solving the Spalart and Allmaras (S-A) equation [20] in the whole field,

$$
\frac{\partial}{\partial t} \int_{\Omega} \tilde{v} d \Omega+\int_{\partial \Omega}\left[\tilde{v} u_{n}-\left(\frac{1}{\sigma_{\tilde{v}}}\left(v_{L}+\tilde{v}\right) \nabla \tilde{v}\right) \cdot \mathbf{n}\right] d S=\int_{\partial \Omega} Q^{t u r} d S
$$

with the source term,

$$
Q^{t u r}=\left[C_{b 1}\left(1-f_{t 2}\right) \tilde{S}\right] \tilde{v}+\frac{1}{\sigma_{\tilde{v}}} C_{b 2}(\nabla \tilde{v})^{2}-\left(C_{w 1} f_{w}-\frac{C_{b 1}}{\varphi^{2}} f_{t 2}\right)\left(\frac{\tilde{v}}{d}\right)^{2} .
$$

The basic idea for DES is to separate the region of RANS and LES by modifying the distance function in Eq. (9) as,

$$
\tilde{d}=\min \left(C_{D E S} \Delta, d\right)
$$

where $C_{D E S}=0.65, d$ is the minimum distance to the wall and $\Delta=\max (\Delta x, \Delta y$, $\Delta z$ ) is the largest distance between the cell center under consideration and the cell center of the neighbors.

The coefficients in these equations are chosen as,

$$
C_{b 1}=0.1335, \quad C_{b 2}=0.622, \quad \sigma_{\tilde{v}}=2 / 3, \quad C_{v 1}=7.1,
$$

and

$$
C_{w 1}=C_{b 1} / \varphi^{2}+\left(1+C_{b 2}\right) / \sigma_{\tilde{v}}, \quad \varphi=0.41
$$

\section{Numerical Discretization}

To solve Eq. (3), the dual-time technique is used by solving the equivalent equations [25],

$$
\partial_{t} \int_{\Omega} \mathbf{U} d V+\frac{\partial \mathbf{U}}{\partial \mathbf{W}} \partial_{\tau} \int_{\Omega} \mathbf{W} d V+\int_{S} \mathbf{F} d S=0
$$

where $\mathbf{W}$ is the vector of primitive variables $(p \mathbf{u} T)^{T}$, and $\partial \mathbf{U} / \partial \mathbf{W}$ is the Jacobian matrix

$$
\frac{\partial \mathbf{U}}{\partial \mathbf{W}}=\left[\begin{array}{ccccc}
\rho_{p} & 0 & 0 & 0 & \rho_{T} \\
u_{1} \rho_{p} & \rho & 0 & 0 & \rho_{T} u_{1} \\
u_{2} \rho_{p} & 0 & \rho & 0 & \rho_{T} u_{2} \\
u_{3} \rho_{p} & 0 & 0 & \rho & \rho_{T} u_{3} \\
\rho_{p} H-\left(1-\rho H_{p}\right) & \rho u_{1} & \rho u_{2} & \rho u_{3} & \rho_{T} H+\rho H_{T}
\end{array}\right] .
$$

In each physical time step, we solve the equation until it reaches a steady state where the term $\partial_{\tau} \int_{\Omega} \mathbf{W} d V$ could be neglected. Equation (13) can be easily discretized at each cell $c$ by the multistage Runge-Kutta schemes, 


$$
\begin{aligned}
& \mathbf{W}_{c}^{(0)}=\mathbf{W}_{c}^{m-1} \\
& \ldots \\
& \mathbf{W}_{c}^{(i)}=\mathbf{W}_{c}^{(0)}-\alpha_{i} \frac{\Delta \tau}{V^{n}}\left(1+\frac{\Delta \tau}{\Delta t}\right)^{-1}\left(\frac{\partial \mathbf{W}}{\partial \mathbf{U}}\right)_{c}^{(i-1)} \operatorname{Res}_{c}^{(i-1)}, \\
& \quad i=1, \ldots, p-1 \\
& \quad \quad \quad \quad \mathbf{W}_{c}^{m}=\mathbf{W}_{c}^{(p)}
\end{aligned}
$$

Here, $\alpha_{i}$ is the coefficient for the $i$-th stage, $V_{c}$ is the cell's volume, $\Delta \tau$ is the pseudo time step, $\Delta t$ is the physical time step, $A_{f}$ is the area of the face $f$ and $\operatorname{Res}_{c}$ is the residue defined as

$$
\operatorname{Res}_{c}=\sum_{f} \mathbf{F}_{f}\left(\mathbf{W}^{-}, \mathbf{W}^{+}, \mathbf{n}\right) \cdot A_{f}+\frac{\mathbf{U}_{c}^{i-1} V_{c}^{n}-\mathbf{U}_{c}^{n-1} V_{c}^{n-1}}{\Delta t} .
$$

The numerical flux $\mathbf{F}_{f}\left(\mathbf{W}^{-}, \mathbf{W}^{+}, \mathbf{n}\right)$ is a function of the extrapolated left-side $(-)$ and right-side $(+)$ values of the face $f$. For example, the numerical flux of ROE scheme $[16,25]$ reads

$$
\mathbf{F}_{f}^{R O E}\left(\mathbf{W}^{-}, \mathbf{W}^{+}, \mathbf{n}\right)=\frac{1}{2}\left[\mathbf{F}\left(\mathbf{W}^{+}, \mathbf{n}\right)+\mathbf{F}\left(\mathbf{W}^{-}, \mathbf{n}\right)-\operatorname{Dis}\left(\mathbf{W}^{-}, \mathbf{W}^{+}, \mathbf{n}\right)\right] .
$$

For all-speed flows, the dissipation term of ROE scheme is scaled and expressed in the following form,

$$
\operatorname{Dis}\left(\mathbf{W}^{-}, \mathbf{W}^{+}, \mathbf{n}\right)=\left|\tilde{u}_{n}^{b}\right| \Delta\left(\begin{array}{c}
\rho \\
\rho \mathbf{u} \\
E
\end{array}\right)+\tilde{\rho} \delta \tilde{u}_{n}^{b}\left(\begin{array}{c}
1 \\
\tilde{\mathbf{u}} \\
\tilde{H}
\end{array}\right)+\delta p\left(\begin{array}{c}
0 \\
\mathbf{n} \\
\tilde{u}_{n}^{b}
\end{array}\right)
$$

with

$$
\begin{gathered}
\delta \tilde{u}_{n}^{b}=\tilde{M}^{*} \Delta u_{n}^{b}+\left[\tilde{a}^{*}-(1-2 \alpha)\left|\tilde{u}_{n}^{b}\right|-\alpha \tilde{u}_{n}^{b} M^{*}\right] \frac{\Delta p}{\tilde{\rho} \tilde{u}_{r e f}^{2}} \\
\delta p=\tilde{M}^{*} \Delta p+\left(\tilde{a}^{*}-\left|\tilde{u}_{n}^{b}\right|+\alpha \tilde{u}_{n}^{b} \tilde{M}^{*}\right) \tilde{\rho} \Delta u_{n}^{b} \\
\tilde{M}^{*}=\frac{\left|\tilde{u}_{\mathbf{n}}^{\prime}+\tilde{a}^{\prime}\right|-\left|\tilde{u}_{\mathbf{n}}^{\prime}-\tilde{a}^{\prime}\right|}{2 \tilde{a}^{\prime}}, \quad \tilde{a}^{*}=\frac{\left|\tilde{u}_{\mathbf{n}}^{\prime}+\tilde{a}^{\prime}\right|+\left|\tilde{u}_{\mathbf{n}}^{\prime}-\tilde{a}^{\prime}\right|}{2}, \\
\Delta(\cdot)=(\cdot)^{+}-(\cdot)^{-}, \quad \tilde{u}_{n}^{b}=\tilde{\mathbf{u}} \cdot \mathbf{n} .
\end{gathered}
$$

The preconditioned velocity in Eq. (21) is

$$
\tilde{u}_{\mathbf{n}}^{\prime}=\tilde{u}_{n}^{b}(1-\alpha)
$$

with

$$
\alpha=\left(1-\beta u_{r e f}^{2}\right) / 2, \quad \beta=\rho_{p}+\rho_{T} /\left(\rho c_{p}\right) .
$$


However, the preconditioned all-speed ROE scheme is unstable and the accuracy may be deteriorated in low-speed flow regions since the reference velocity in Eq. (19) is used and defined as

$$
u_{\text {ref }}=\min \left[a, \max \left(|\mathbf{u}|, K\left|\mathbf{u}_{\infty}\right|\right)\right]
$$

where $\mathbf{u}$ is the local velocity, $\mathbf{u}_{\infty}$ is a fixed reference speed (it is usually set to be free stream velocity), and $a$ is the local sound speed, $\mathrm{K}$ is a constant (which is chosen as $0.5)$.

In order to consider the low speed flows as well as preserve the accuracy, the SLAU (Simple Low dissipation AUSM) [18] is employed. It is simple and does not need the "cutoff Mach number" that other all-speed schemes (ex., ROE) have. Hence, it can be applied to any low Mach number problem without further problem dependent adjustments. The numerical flux $\mathbf{F}_{f}\left(\mathbf{W}^{-}, \mathbf{W}^{+}, \mathbf{n}\right)$ for SLAU is defined as

$$
\mathbf{F}_{f}^{S L A U}\left(\mathbf{W}^{-}, \mathbf{W}^{+}, \mathbf{n}\right)=\frac{1}{2}\left[(\dot{m}+|\dot{m}|) \Upsilon^{-}+(\dot{m}-|\dot{m}|) \Upsilon^{+}\right]+p_{f} b_{n}
$$

with

$$
\Upsilon=\left(\begin{array}{c}
1 \\
\mathbf{u} \\
H
\end{array}\right) \quad \text { and } \quad b_{n}=\left(\begin{array}{l}
0 \\
\mathbf{n} \\
0
\end{array}\right) .
$$

The mass flow rate and the pressure diffusion term are

$$
\dot{m}=\rho^{-}\left(u_{n}^{-}+q^{-}\right)+\rho^{+}\left(u_{n}^{+}-q^{+}\right)-x\left(p^{+}-p^{-}\right) / a_{f},
$$

and

$$
p_{f}=\left(\beta^{+}-\beta^{-}\right)\left(p^{-}-p^{+}\right)+\left[(1-x)\left(\beta^{+}+\beta^{-}-1\right)+1\right]\left(p^{-}+p^{+}\right)
$$

with

$$
\beta^{+}=\beta^{+}\left(M_{n}^{-}\right), \quad \beta^{-}=\beta^{-}\left(M_{n}^{+}\right), \quad M_{n}^{k}=u_{n}^{k} / a_{f}, \quad k=-,+.
$$

The interface sound speed $a_{f}$ and the split Mach number polynomials are defined as

$$
a_{f}=\left(a^{-}+a^{+}\right) / 2,
$$

and

$$
\beta^{ \pm}(M)= \begin{cases}\frac{(M \pm 1)^{2}(2 \mp M)}{4}, & \text { if }|M|<1 \\ \frac{M \pm|M|}{2 M}, & \text { else }\end{cases}
$$

respectively.

The other functions are defined as follows

$$
\begin{gathered}
q^{k}=(1-g)\left|\bar{u}_{n}\right|+g\left|u_{n}^{k}\right|, \quad k=-,+, \\
g=-\max \left[\min \left(M_{n}^{-}, 0\right),-1\right] \cdot \min \left[\max \left(M_{n}^{+}, 0\right), 1\right],
\end{gathered}
$$


Fig. 3 Mesh for low speed flow over a cylinder $(128 \times 33)$

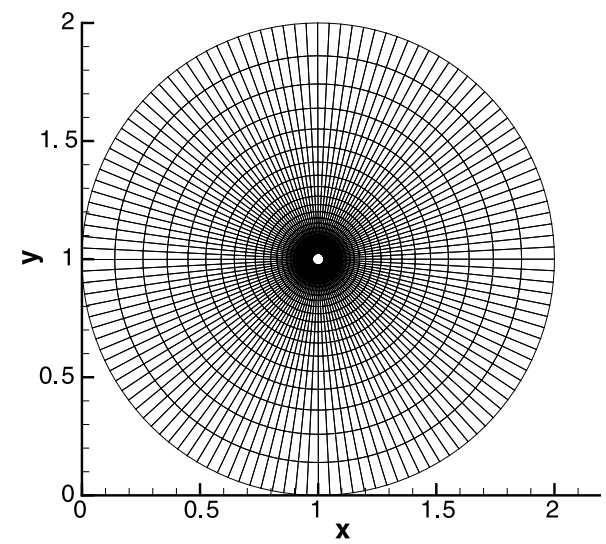

$$
\begin{gathered}
\left|\bar{u}_{n}\right|=\frac{\rho^{-}\left|u_{n}^{-}\right|+\rho^{+}\left|u_{n}^{+}\right|}{\rho^{-}+\rho^{+}}, \\
x=(1-\hat{M})^{2}, \quad \hat{M}=\min \left[1,1 / a_{f} \sqrt{\left(\mathbf{u}^{-2}+\mathbf{u}^{+2}\right) / 2}\right] .
\end{gathered}
$$

In order to evaluate the two different schemes and choose which one to use, the comparisons of the pressure contours between the ROE scheme and the SLAU scheme for the 2D in-viscid low speed flow $(\mathrm{Ma}=0.01)$ over a cylinder on the $\mathrm{O}$ type mesh (Fig. 3) are presented in Fig. 4. It is clear that the ROE scheme does not perform well in this case because of the strong asymmetric nature of the pressure contours. Whereas, the SLAU scheme shows symmetric contours as expected, so we opt for this scheme in this paper.

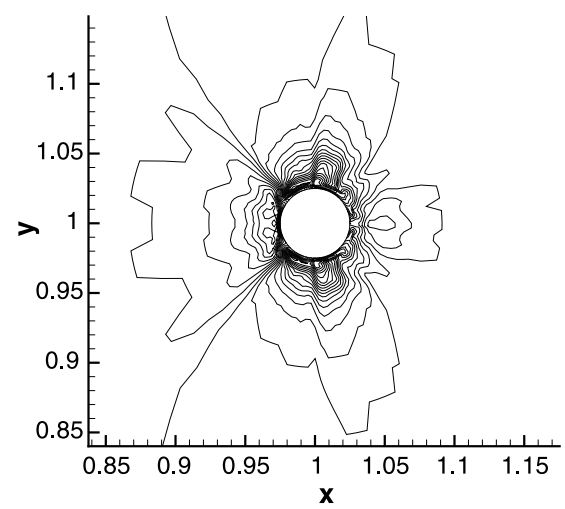

(a) ROE scheme

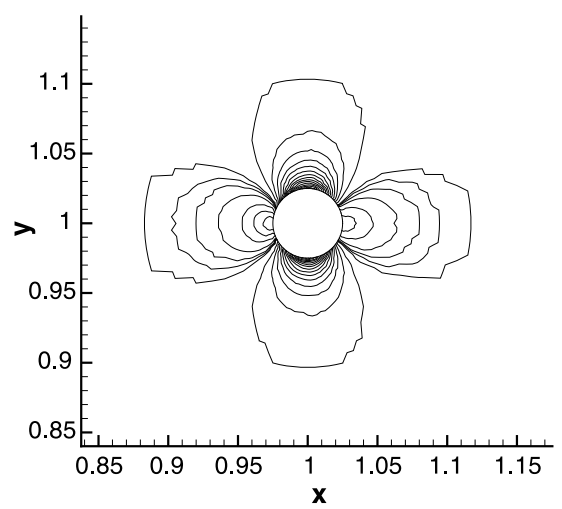

(b) SLAU scheme

Fig. 4 Pressure contours for the low speed flow $(\mathrm{Ma}=0.01)$ over cylinder 
Fig. 5 Mesh

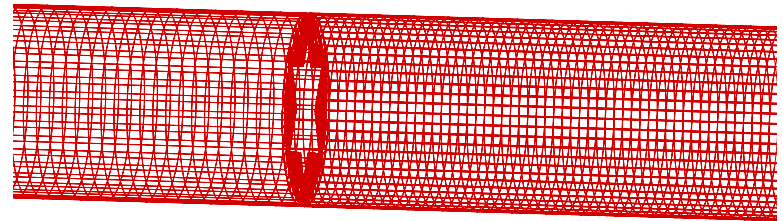

\section{Results}

In order to assess the ability of our density-based DES code to predict a subsonic turbulent flow after a fractal orifice, turbulent pipe flows forced through a multiscale fractal-shaped orifice are investigated. A fractal shape is a geometrical shape where an identical pattern repeats itself at different scales. Here, a snowflake fractal shape with two iterations (SF2) is used to generate the orifice as illustrated in Fig. 2. Its construction begins with a triangle of unit length 1 , then the middle third of each side is removed and replaced with two lines each having the same length $(1 / 3)$. By repeating this procedure twice, the snowflake fractal shape (SF2) will appear.

The experimental set up is described in more details in [1]. It consists of a wind tunnel which is composed of two Perspex tubes, each two meter long (Fig. 1). The two Perspex tubes have the same outer and inner diameters, respectively $150 \mathrm{~mm}$ and $140 \mathrm{~mm}$. A bell-mouth is put at the inlet of the first Perspex tube and the fan motor is connected to the end of the other Perspex tube via a T-junction. The flow is controlled in the pipe through an outlet rotating valve placed at the exit of the Perspex pipe near the fan. The wind tunnel uses a "pull method" of drawing air from the pipe with a valve to vary the velocity in the wind tunnel. The fractalshaped orifice is put after the center position $0.22 \mathrm{~m}$ toward the outlet. Velocity time series are obtained using hotwire anemometry. The measurements are taken through different holes made on the pipe wall the first one at $70 \mathrm{~mm}$ and then every $35 \mathrm{~mm}$ from the fractal grid (see Fig. 1). At a given hole measurements are recorded at different distances from the wall. This allows us to derive profiles of the different velocity statistics against which the numerical results can be validated.

To investigate the performance of the present solver for turbulence flows generated by a fractal-shaped orifice, we run this case in our local cluster Bluegrid. The mesh consists of 511908 cells as shown in Fig. 5. The inlet velocity is set to $9.4 \mathrm{~m} \mathrm{~s}^{-1}$. The mean velocity profile is a good indicator of the structure of the turbulent flow. The experimental mean velocity profiles were obtained by calculating the mean velocity at different points over a long time. To compare with the experimental results, we export the data at the sections corresponding to the experimental results. Note that we compare the same quantity which is the average absolute velocity (the only one a single hotwire can measure).

The comparisons of the mean velocity profiles are conducted from hole 1 to hole 7. The results are shown in Figs. 6-9. Figures 6 and 7 compare the mean velocity profiles at the locations close to the orifice. The peak value and the shape are very close to the experiment results although the tip of the profile does not match the experimental result exactly. It also seems that the measurements show less suction 
Fig. 6 Mean velocity profile at hole 1



velocity at the position which is closed to the wall. Discrepancy between the computations and the experiment near the wall is less surprising if one remembers that this is where the experimental results are the less accurate and the single hotwire technique does not work properly. One has also to bear in mind that it is the average absolute velocity that is shown so its convergence to zero near the wall is slower than that of the velocity itself. However, that is one issue with DES that need to be worked on.

Figures 8 and 9 compare the mean velocity profiles at holes 4 to 7 which are a little further from the orifice. This time computations agree reasonably well with the experiment. It could also be easily observed that as expected the profile is influenced by the fractal shape at the positions which are close to the fractal orifice (Figs. 6 and 7).

Note that in our simulation the geometry is quite complex as the thickness of the fractal-shaped orifice is very small $(0.003 \mathrm{~m})$ when compared to the whole length of the pipe $(4 \mathrm{~m})$. Hence, an unstructured mesh (with 127520 Brick cells, 360956 Tetra cells, and 23432 Pyramid cells) has to be used and unfortunately, such mesh
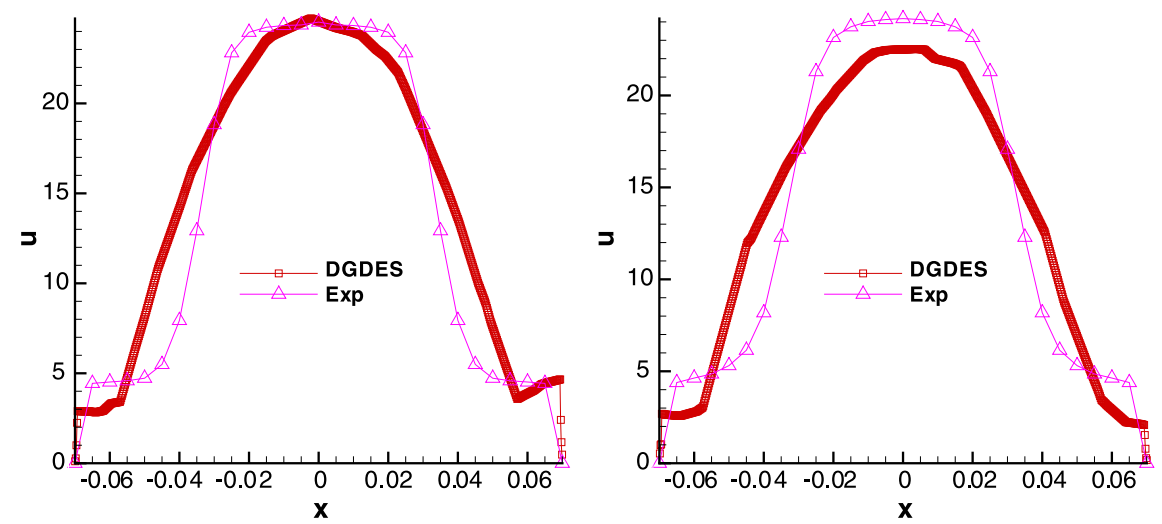

Fig. 7 Mean velocity profile at hole 2, left and hole 3, right 

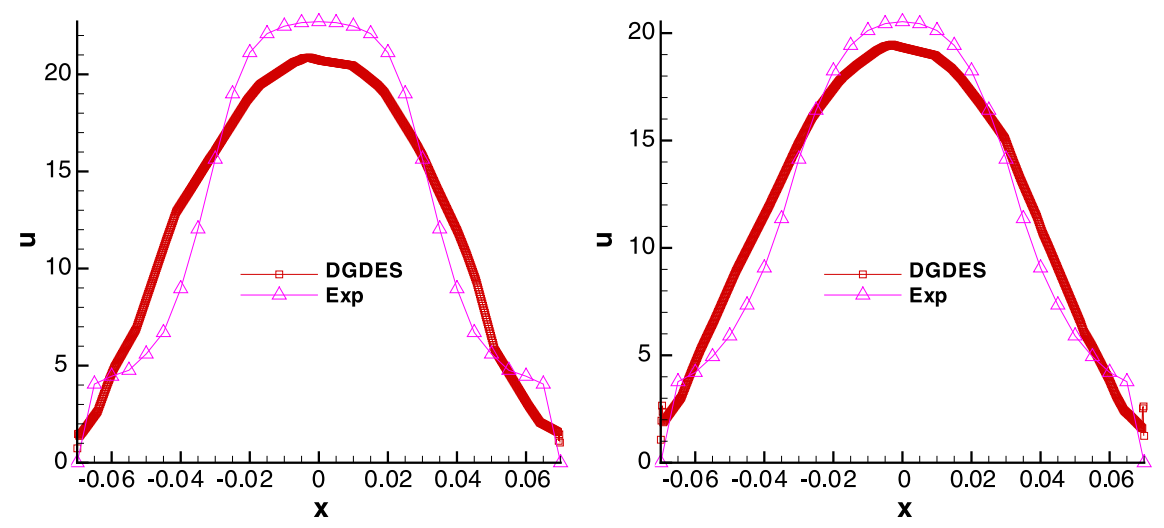

Fig. 8 Mean velocity profile at hole 4, left and hole 5, right
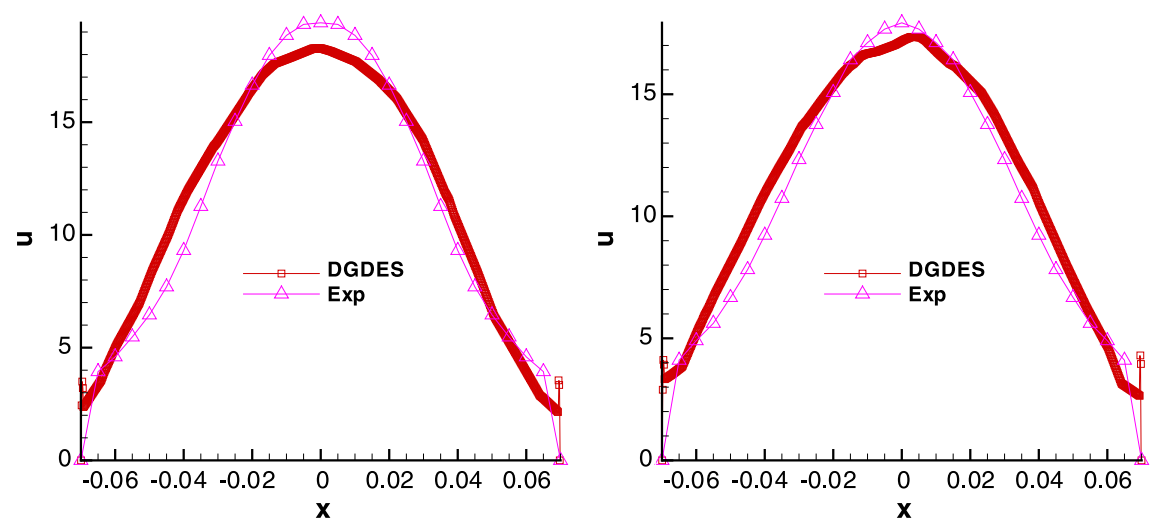

Fig. 9 Mean velocity profile at hole 6, left and hole 7, right

can not be guaranteed to be symmetric, especially in the regions which are closed to the fractal orifice. This explains why the profiles at the holes which are closed to the fractal are not fully symmetric. This is part of the problems that need to be solved for using DES. It shows how complicated the flow is near the fractal orifice.

\section{Conclusions}

In this paper, the turbulent pipe flow through a snow flake fractal shape orifice (SF2) is investigated by the detached eddy simulation method (DES). In order to tackle the low speed problem by the density based method, the SLAU (Simple Low dissipation AUSM) [18] is adopted in this paper. Owing to the low numerical dissipation in low speeds, the scheme performs well for these speeds when compared to the ROE scheme. The results for the turbulent flows after the orifice are compared with 
those of the corresponding experiment [1, 3]. They show good agreements in the mean velocity profiles at different holes. Results need to be improved near the walls and near the fractal orifice. However, there is scope for using DES to study more iterations of the fractal grid.

Acknowledgements Fruitful discussions with J.C. Vassilicos and S. Laizet are gratefully acknowledged.

This work was supported by the Engineering and Physical Sciences Research Council through the UK Turbulence Consortium (Grant EP/G069581/1).

\section{References}

1. Abou El-Azm Aly, A., Chong, C.H.A., Nicolleau, F.C.G.A., Beck, S.B.M.: Experimental study of the pressure drop after fractal-shaped orifices in a turbulent flow pipe. Proc. World Acad. Sci., Exp. Therm. Fluid Sci. 34, 104-111 (2010)

2. Chester, S., Meneveau, C., Parlange, M.B.: Modeling turbulent flow over fractal trees with renormalized numerical simulation. J. Comput. Phys. 225, 427-448 (2007)

3. Chong, A.C.H.: Turbulence flow generated by a fractal grid. PhD thesis, The University of Sheffield (2008)

4. Davila, J., Vassilicos, J.C.: Richardson pair diffusion and the stagnation point structure of turbulence. Phys. Rev. Lett. 91, 144-501 (2003)

5. Fung, J.C.H., Vassilicos, J.C.: Two-particle dispersion in turbulentlike flows. Phys. Rev. E 57(2), 1677-1690 (1998)

6. Goto, S., Osborne, D.R., Vassilicos, J.C., Haigh, J.D.: Acceleration statistics as measures of statistical persistence of streamlines in isotropic turbulence. Phys. Rev. E 71, 015301(R) (2005)

7. Hurst, D., Vassilicos, J.C.: Scalings and decay of fractal-generated turbulence. Phys. Fluids 19(3), 035103 (2007)

8. Karypis, G., Kumar, V.: User manual of METIS: a software package for partitioning unstructured graphs, partitioning meshes and computing fill-reduced orderings of sparse matrices, version 4.0. University of Minnesota (1998)

9. Laizet, S., Vassilicos, J.C.: Multiscale of turbulence. J. Multiscale Model. 1(1), 177196 (2009)

10. Laizet, S., Lamballais, E., Vassilicos, J.C.: A numerical strategy to combine high-order schemes, complex geometry and massively parallel computing for the DNS of fractal generated turbulence. Comput. Fluids 39(3), 471-484 (2010)

11. Mandelbrot, B.: On the geometry of homogeneous turbulence with stress on the fractal dimension of the iso-surfaces of scalars. J. Fluid Mech. 72(2), 401-416 (1975)

12. Mazzi, B., Vassilicos, J.C.: Fractal-generated turbulence. J. Fluid Mech. 502, 65-87 (2004)

13. Mazzi, B., Okkels, F., Vassilicos, J.C.: A shell-model approach to fractal-induced turbulence. Eur. Phys. J. B 28, 243-251 (2002)

14. Meneveau, C., Sreenivasan, K.R.: Interface dimension in intermittent turbulence. Phys. Rev. A 41(4), 2246-2248 (1990)

15. Queiros-Conde, D., Vassilicos, J.C.: Intermittency in Turbulence and Other Dynamical Systems, Turbulent Wakes of 3-D Fractal Grids. Cambridge University Press, Cambridge (2001)

16. Roe, P.L.: Approximate Riemann solvers, parameters vectors and difference schemes. J. Comput. Phys. 43, 357-372 (1981)

17. Seoud, R.E., Vassilicos, J.C.: Dissipation and decay of fractal-generated turbulence. Phys. Fluids 19(10), 105108 (2007)

18. Shima, E., Kitamura, K.: On new simple low-dissipation scheme of AUSM-family for all speeds. In: 47th AIAA Aerospace Sciences Meeting Including The New Horizons Forum and Aerospace Exposition, AIAA 2009-136 (2009) 
19. Spalart, P.R.: Young-Person's guide to detached-eddy simulation grids. Technical Report NASA/CR-2001-211032, NASA Langley Research Center, Hampton, VA (2001)

20. Spalart, P.R., Allmaras, S.R.: A one-equation turbulence model for aerodynamic flows. AIAA Paper 92-0439 (1992)

21. Spalart, P.R., Jou, W.-H., Strelets, M., Allmaras, S.R.: Comments on the feasibility of LES for wings, and on a hybrid RANS/LES approach. In: Advances in DNS/LES (Proceedings of 1st AFOSR Int. Conf. on DNS and LES, Louisiana Tech.), pp. 137-147. Greyden Press, Dayton (1997)

22. Sreenivasan, K.R., Meneveau, C.: The fractal facets of turbulence. J. Fluid Mech. 173, 357386 (1986)

23. Sreenivasan, K.R., Ramshankar, R., Meneveau, C.: Mixing, entrainment and fractal dimensions of surfaces in turbulent flows. Proc. R. Soc. Lond. A 421, 79-108 (1989)

24. Vassilicos, J.C., Fung, J.C.H.: The self-similar topology of passive interfaces advected by twodimensional turbulent-like flows. Phys. Fluids 7(8), 1970 (1995)

25. Weiss, M., Smith, W.A.: Preconditioning applied to variable and constant density flow. AIAA J. 33, 2050-2057 (1995)

26. Xia, H.: Dynamic grid detached-eddy simulations for synthetic jet flows. PhD thesis, The University of Sheffield (2005)

27. Xia, H., Qin, N.: Dynamic grid and unsteady boundary conditions for synthetic jet flow. AIAA 2005-106 (2005) 